\title{
INTR ACELLULAR LOCALIZATION AND CO-FACTOR REQUIREMENT OF NITROREDUCTASE IN RAT LIVER
}

\author{
RYUICHI KATO* AND 'TAKAO OSHIMA** \\ * Department of Pharmacology, National Institule of Hygienic Sciences, Selagaya-ku, Tokvo \\ **Deparlment of Pharmacology, School of Medicine, Keio University, Shinjuku-ku, Tokyo \\ Rereived for publication June 17, 1968
}

Numerous aromatic nitrocompounds are reduced to aromatic amines by mammalian liver. Fiouts and Brodie (1) reported that the nitroreductase was localizer in 9.000) $\times \mathrm{g}$ supernatant of liver. Although both $\mathrm{NADPH}$ and $\mathrm{NADH}$ can serve as electron donors, $\mathrm{NADPH}$ was abrut four times more cffective than $\mathrm{NAl} O H$. It is known that hydroxylamines are formed as intermediate in the reduction of nitrocompounds to aromatic amines (1, 2). However, many works on the reduction of nitrocompounds were carried out by measuring lie formation of aromatic amines (1 3).

In the present studies, therefore, the step of nitroreduction was separated into twe steps and the intracellular localization and co-factor requirement were investigated.

Male rats of Wistar strain, weighing about $170-200 \mathrm{~g}$ were used. The microsomes and supernatant were preparated and the incubation was carried out in atomosphere of nitrogen as described in previous paper (4). $p$-Nitrobenzoic acid (NBA) and p-hydroxyaminobenzoic acid (HABA) were used as the substrate. $p$ Hydroxyaminobenzoic acid was synthesized according to the method of Bauer and Rosenthal (5). Determination of p-nitrobenzoic acid was carried out spectrophotometrically and p-hydroxyaminobenzoic acid was

TABLE 1. Intracellular localization and co-factor requirement of the reduction of $p$-nitrobenzoic acid and $p$-hydroxyaminobenzoic acid by rat liver.

\begin{tabular}{llccc}
\hline Cell fraction & Co-factor & \multicolumn{2}{c}{$p$-Nitrobenzoic acid } & $p$-Hydroxyaminobenzoic acid \\
& & HABA formed & ABA formed & ABA formed \\
Microsomes & NADPH & $0.86+0.12$ & $0.79 \div 0.05$ & $1.64: 0.15$ \\
& NADH & $0.08 \div 0.02$ & $0.03 \div 0.01$ & $1.14-0.08$ \\
Supernatant & NADPH & $0.81-0.05$ & $0.08 \div 0.02$ & $0.57 \div 0.04$ \\
& NADH & $0.76 \div 0.06$ & $0.07 \div 0.01$ & 0.50 \\
Microsomes $:-$ & NADPH & $1.38 \div 0.14$ & $0.95 \div 0.08$ & $2.32 \div 0.21$ \\
Supernatant & NADH & $0.97 \div 0.10$ & $0.50 \div 0.03$ & $1.55+0.11$ \\
\hline
\end{tabular}

The activities are expressed by / mole per $\mathrm{g}$ wet weight of liver per 30 minutes and given as the average : standard error from 34 experiments. The incubation mixture contained the followings in total volumes of $5 \mathrm{ml} ; 2 \mathrm{ml}$ of tissue preparation equivalent to $500 \mathrm{mg}$ of liver, 0.8 /mole NADP or NAD, 20 \%mole G-6-P or 10) "tmole alcohol, 25 imole $\mathrm{MgCl}_{2}, 50$, acid or p-hydroxyaminobenzoic acid, $1.4 \mathrm{ml}$ of $0.2 \mathrm{~m}$ phosphate buffer $(\mathrm{pH} \mathrm{7.4)}$ and $1.0 \mathrm{ml}$ of $1.15 \%$ KCi. G-6-P dehydrogenase (0.5 U) or alcohol dehydrogenase (2.0 U) was added when the microsomes were used. The incubation was run in duplicate at $37 \mathrm{C}$ under nitrogen phase for 30. minules.

HABA : p-hydroxyaminobenzoic acid, ABA : p-aminobenzoic acid. 
determined by the chelate formation with pentacyanoaquaferroate (6). p-Aminobenzoic acid (ABA) was determined by the method of Fouts and Brodie (1).

Table 1 shows that NADPH-linked reduction of $p$-nitrobenzoic acid to $p$-hydroxyaminobenzoic acid is mainly localized in microsomes, whereas NADH-linked nitroreduction is localized in supernatant. The activity of NADPH- and NADH-linked nitroreduction is almost same in supernatant. Moreover, the reduction of $p$-hydroxyaminobenzoic acid is done in both microsomes and supernatant, but the activity is higher in microsomes than that in supernatant.

The NADPH-linked activity is about $50_{\%}^{\circ}$ higher than the NADH-linked activity in microsomes. Thus, the NADH-linked reduction of $p$-nitrobenzoic acid to $p$-aminobenzoic acid is negligible in microsomes, but the NADH-linked reduction in microsomes + supernatant is about $30 \%$ of the NADPH-linked activity in accord with results of Fouts and Brodie (1). These results suggested that the first step of the NADH-linked reduction of $p$-nitrobenzoic acid to $p$-aminobenzoic acid is mainly done by supernatant and the second step is mainly mediated by microsomes. The NADPH-linked reduction of $p$-nitrobenzoic acid to $p$-hydroxyaminobenzoic acid is the important step for all over nitroreduction and this step is likely the rate limiting step.

In further experiments it was shown that the NADPH-linked reduction of $p$-nitrobenzoic acid to $p$ hydroxyaminobenzoir arid was inhibited by oxygen and stimulated by FMN or FAD and the microsomal activity was stimulated by phenobarbital. The NADPH-linked nitroreductase of microsomal fraction likely differed from that of supernatant, because the former was stimulated by phenobarbital and inhibited by oxygen, whereas the later was not stimulated by phenobarbital and only partially inhibited by oxygen. The detailed results will be published elsewhere (7).

\title{
REFERENC'ES
}

1) Fouts, J.R. and Bronil, B.B.: J. Pharmac. exp. Ther. 119, 197 (1957); 2) КАмm, J.J. And Giflift'te, J.R.: Life Sci. 2, 259 (1963); 3) Hart, L.G., Adamson, R.H., Dixon, R.I. and Fouts, J.R.: J. Pharmac. exp. Ther. 137, 103 (1962); 4) Kato, R. and Takahashi, A.: Mol. Pharmac. 4, lo9 (1968); 5) Baver, H. and Rosevtral, S.M.: J. Chem. Soc. 6, 611 (1944); 6) Feigle, F.: Spot Test in Organic Analysis, Vol. 2, p. 121, Elsevier Pub. Comp., Amsterdam (1954); 7) Kato, R., Oshrma, T. AND TAKANAKA, $A .:$ in preparation

\section{ANTITREMOR ACTION OF A NEW BETA RECEPTOR BLOGKING AGENT (I.C.I. 50, 172)}

\author{
G. ACHARI AND S.P. SINHA \\ Department of Pharmacology, P. W. Medical College, Patna-4, India
}

Received for publication June 18, 1968

In a previous communication (1) it was reported that propranolol (Inderal) a beta receptor blocking agent abolishes tremor induced by tremorine. A number of structurally similar adrenergic beta receptor blocking agents have been synthesized recently and their actions are almost similar qualitatively in that they all block beta receptor but they differ quantitatively. A new beta receptor blocking agent (I.C.I.50, 172), which does not block all the receptors (2) have been studied in rats to find out the action if tremorine (1,4-dipyrdino2-butyne) induced tremor and its cholinergic effects are blocked by this new beta receptor blocking agent I.C.I. $50,172$.

Methods: Experiments were carried out in albino rats (C.D.R.I., Strain) of both sexes weighing between 